\title{
Effects of Eimeria Species on Different Rabbit Breeds
}

\author{
Karina V. Sidorenko, Manya E. Mkrtchyan*, Marina S. Petrova and Elmira N. Taimusova \\ St. Petersburg State University of Veterinary Medicine, St. Petersburg City, Russia. \\ Corresponding author email: Smolentsev82@mail.ru
}

\section{ABSTRACT}

A limiting factor in the successful development of rabbit farming is diseases of invasive etiology, in particular eimerioses. The main reason for the underproduction is the massive destruction of the cells of the intestinal mucosa. Studies were carried out on Californian (C), Soviet Chinchilla (S) rabbits and their hybrids at 30, 45 and 60 days of age. A group of 6 animals in each of the experimental (infested) and control (intact) groups was formed. One-day-old rabbits of the experimental groups were infected with different doses of oocysts (50,000, 100,000 or 200,000 per individual) of the Eimeria species E. perforans and E. irresidua. On days 30, 45 and 60 after infection, animals in the experimental and control groups were slaughtered to assess the intensity of infection. Material was selected for histological examination. Various staining methods were used to contrast endogenous stages of Eimeria in the intestinal mucosa: alcian blue and Carazzi haematoxylin; Mayer's haematoxylin and 1\% alcohol eosin; Gill's haematoxylin and 1\% alcohol eosin; Van Gieson picro-fuchsin; toluidine blue. When determining the intensity of infestation in different breed and age groups, it was found that Californian animals were most susceptible to infestation by E. perforans and E. irresidua oocysts, regardless of the dose of infestation. The histological examination of the small intestine of infected rabbits of experimental breedage groups revealed that despite the absence of any clinical manifestations, damage to the epithelial plate of the small intestine mucosa was found in the epithelial plate of the intestinal villi as well as significant damage in the intrinsic plate of the mucosa, which are most effectively revealed by staining with alcyan blue and Carazzi hematoxylin and toluidine blue.

KEY WORDS: RABBITS, BREEDS, INTENSITY OF INFESTATION, ALCIAN BLUE, CARAZZI'S HAEMATOXYLIN, TOLUIDINE BLUE.

\section{INTRODUCTION}

In order to provide the public with livestock products, there are many options for the production and breeding of rabbits, which is a rather attractive way of obtaining the required volume of meat, high quality pelts and also laboratory animals for research activities. A limiting factor in the successful development of rabbit farming in the world, and in particular in the Russian Federation, is diseases of invasive etiology. The causative agents of these diseases have evolutionarily adapted to parasitise in animals. The pathological processes recorded in parasitic diseases are influenced by certain factors,

Biosc Biotech Res Comm P-ISSN: 0974-6455 E-ISSN: 2321-4007

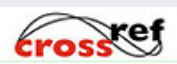

Identifiers and Pagination

Year: 2021 Vol: 14 No (9) Special Issue

Pages: 235-239

This is an open access article under Creative

Commons License Attribn 4.0 Intl (CC-BY). DOI: $h t t p: / / d x$.doi.org/10.21786/bbrc/14.9.44

including: the animal type, its breed, type of feeding and housing, hygiene, immune status; parasite specificity, their ability to adapt, including competitiveness through rapid reproduction rates; presence of factors causing the pathogenic properties of parasites, etc (Edwards et al., 2016).

The animal organism reacts to the penetration of the pathogen with a complex of pathophysiological and immunomorphological reactions. The defence systems of the affected organism focus on eliminating the pathogen and repairing the structural and functional damage inflicted during the infestation. The range of effects of parasites on the host is extremely wide, and often the same parasite can act in different ways. The criterion for the degree of pathogenic effect on the host is the concept of virulence (Dimitrijevic et al., 2018).

Any disease of invasive etiology causes damage to internal organs as well as to whole organ systems. Clinical and laboratory tests are carried out to determine the exposure of animals to the pathogen. In turn, an autopsy

Article Information

Received: $19^{\text {th }}$ Apr 2021 ccepted after revision: $24^{\text {th }}$ June 2021 
reveals macroscopic changes to internal organs and tissues. The pathomorphological assessment also allows for analysis of the nature of changes in the damaged organs and tissues at the histological level. The results of the experiment will help to develop or adjust the directions of prevention and treatment of sick animals. In this work we considered it important to study the pathomorphological changes occurring in the small intestine of rabbits infected with protozoa of the genus Eimeria (Kuznetsov et al., 2018).

Eimeriosis is one of the main causes of stunted growth and large wasting of young farm animals, reduced live weight as well as the quality of the wool coat of adult animals. The main reason for the underproduction is the massive destruction of the cells of the intestinal mucosa, which is represented by several plates. Its epithelial plate is made up of multiple populations of diverse cells that perform specific functions. The most important of these are the caemic enterocytes, which ensure parietal digestion and absorption of nutrients into the bloodstream. It is these cells that are mainly affected by the endogenous stages of Eimeridae. The intrinsic mucosal plate forms the basis of the intestinal villi, elements that allow a significant increase in the useful area of digestion and absorption, greatly increasing the organ's efficiency in its basic functions.

Members of the Eimeria genus localise in the epithelial and intrinsic plate cells of the intestinal tube mucosa, causing catarrhal haemorrhagic enteritis, manifested by hyperemia and swelling of the mucosa, epithelial desquamation and the intestinal villi disruption. Due to the pathogenic action of the parasite, the surface of the mucous membrane of the organ is covered with a viscous, transparent mucus. Diapedesis haemorrhages occur take place. In some cases, infestations are chronic and asymptomatic, making them difficult to diagnose in a timely manner. (Kuznetsov et al. 2020) note in their work that coccidia have a significant impact on the morphobiological and immunological qualities of mucus, which is important for the vertebrate microbiota. The aim of our research was to compare the pathogenic effects of Eimeria on rabbits of different breeds.

\section{MATERIAL AND METHODS}

Studies were conducted at the Department of Biology, Ecology and Histology, St Petersburg State University of Veterinary Medicine on rabbits of Californian (C), Soviet Chinchilla (S) and their hybrids at 30, 45 and 60 days of age. In the experiment, pairwise comparisons were made between experimental (infested) and control (intact) groups of 6 animals each: purebred $C^{*} \mathrm{C}$, purebred $\mathrm{S}^{*} \mathrm{~S}$, and hybrid $\mathrm{S}^{*} \mathrm{C} / \mathrm{C}^{*} \mathrm{~S}$. On the first day after birth, the rabbits of the experimental groups were infected with different doses of oocysts $(50,000,100,000$ or 200,000 per individual) of the Eimeria species E. perforans and E. irresidua (Fadunsin et al., 2017).

On days 30, 45 and 60 after infection, animals in the experimental and control groups were slaughtered to assess the intensity of infection. Material was selected for histological examination. To determine the intensity of infestation, 3 samples of 1 gram of contents from each section of the intestine were taken and examined by the Fülleborn flotation method, using a vessel with a known surface area. After settling with a parasitological loop with a known area, six drops were taken from the surface of the suspension and transferred onto slides. Oocysts were counted in all six drops.

The arithmetic mean number in one drop was then calculated. The number of oocysts in $1 \mathrm{~g}$ of starting material was calculated from the ratio of the loop area to the surface area of the beaker. Histological examination of $10 \mathrm{~mm} \times 10 \mathrm{~mm}$ sections of the small intestine was carried out according to the protocol for making microslides. The samples were fixed in standardised 10\% formalin for 2 to 4 days. Organ fragments were washed in running tap water for 1 to 2 hours before being wired in a battery of alcohol. This was followed by dewatering using a six-stage isoprep battery and a three-stage sealing in HISTOMIX paraffin medium for 20 minutes each, before being poured into the paraffin medium to form blocks. Sections 4-4.5 $\mu \mathrm{m}$ thick were made on a ROTMIC-2M rotary motorised microtome. Before staining, paraffin was removed by dissolving it in xylene and 96\% ethanol for 4 minutes in each medium and washed in distilled water for 5 minutes (Mykhailiutenko et al., 2019).

Various staining methods were used to contrast endogenous stages of Eimeria in the intestinal mucosa: alcian blue and Carazzi haematoxylin; Mayer's haematoxylin and 1\% alcohol eosin; Gill's haematoxylin and 1\% alcohol eosin; Van Gieson picro-fuchsin; toluidine blue. Histopreparations were examined by light microscopy using MIKMED-5 optics at x 10 eyepiece magnification and $\mathrm{x} 40$ and $\mathrm{x} 100$ objectives. Statistical processing of infestation intensity data was carried out with Past 3 software. The reported study was funded by RFBR project No.19-316-90059. (The reported study was funded by RFBR, project No.19-316-90059)

\section{RESULTS AND DISCUSSION}

When studying the resistance of different rabbit breeds and their hybrids to experimental infection with certain doses of oocysts of Eimeria association, it was found that when infected with 50,000 oocysts, the maximum intensity of infestation one month after infection was observed in californian rabbits (21435.8 \pm 386.1 oocysts), which exceeded the animals of the hybrid and Soviet chinchilla breed groups by $15 \%$ and $9 \%$ respectively. At day 45 of infection, the highest intensity of infestation was observed in californian and hybrid rabbits, which exceeded the average of purebred Soviet chinchilla by $12 \%$ and $17 \%$ respectively. However, on day 60 of infection, the number of oocysts detected in the Californian breed was almost equal to that of the Soviet chinchilla with a difference of 398 oocysts on average, outstripping the hybrid by only $4 \%$.

The following trend can be observed when considering 
the average infestation intensity in different breed and age groups when 100,000 oocysts are infested. Animals of the Californian breed at day 30 and 45 of infection show the greatest susceptibility and parasite establishment, outperforming hybrid rabbits by an average of 20,763 oocysts and 17,859 oocysts, respectively. However, by the end of the experiment the rabbits of the hybrid breed group had the highest rate of infestation $(18918.9 \pm 167.4$ oocysts), 3.6\% more than those of the Californian breed (18236 \pm 284.1 oocysts) (Pehlivanoglu et al., 2016).

Analysing the intensity of infestation at the maximum dose of infestation in experimental rabbits of all breed and age groups, it was found that at day 30 of infection a comparable and almost identical degree of infestation was observed. If you compare it with the Californian breed, the difference is between 3\% and 5\%. On day 45 of infection, the lowest intensity of infestation was found in rabbits of the hybrid group (17583.6 \pm 442.7 oocysts), yielding to purebred animals of the Soviet chinchilla by almost $16 \%$ and to the Californian by about $20 \%$. However, at two months of age in purebred rabbits, the intensity of infestation ranged from an average of $18748.59 \pm 139.7$ to $18996.6 \pm 267.8$ oocysts per head. Hybrid two-month-old rabbits have the lowest rate of infestation, about $17390 \pm 80.2$ oocysts, which is $8.5 \%$ and 7.3\% lower than in the California and Soviet chinchilla breeds, respectively.

Figure 1: Damage caused by endocytic stages of small intestinal mucosa (Stains: alcian blue and Carazzi's haematoxylin; magnification: x400 (A) and x1000 (B)
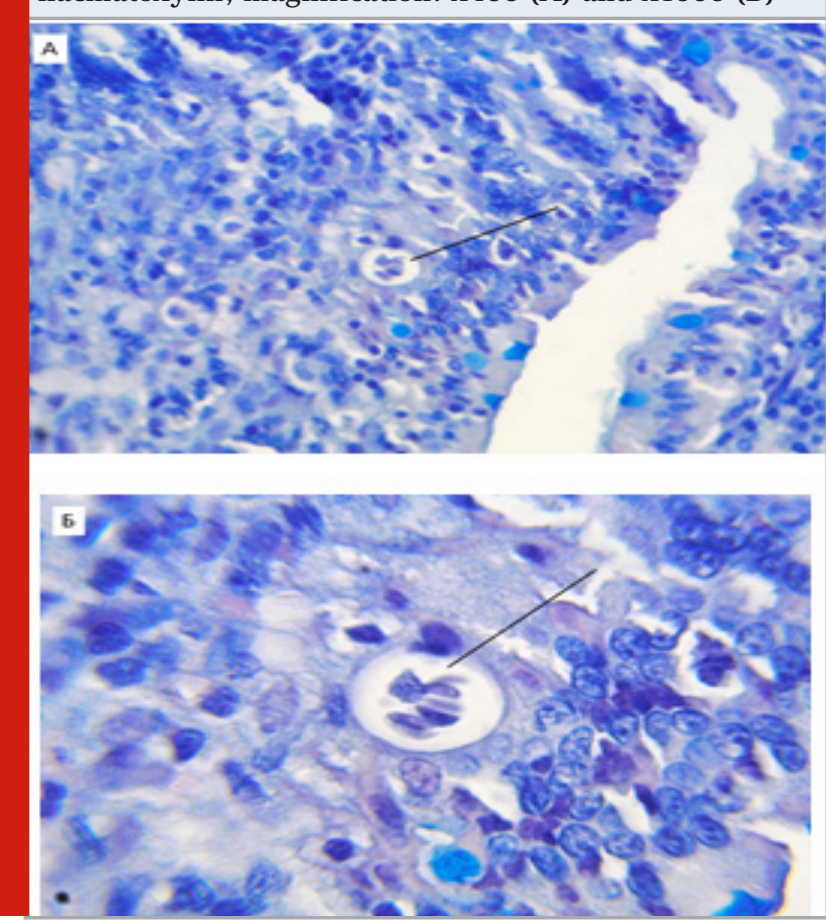

We have also investigated the pathomorphological changes observed in small intestinal wall lesions by endogenous stages of Eimeria association. For better visualisation of microstructures, different staining methods were tried in order to determine the most promising method to study the pathomorphological effects of coccidia on the cellular elements of the intestinal mucosa. It was found experimentally that of the five staining methods studied, not all of them gave a complete and clear picture of the pathological changes.In our opinion, alcian blue and Carazzi's haematoxylin or toluidine blue stains are the most contrasting and facilitate the rapid detection of endocytic forms of Eimeridae. The use of alcyanine blue, on the other hand, allows the detection of secretory bokalovid cells (Figure 1).

Figure 2. Endocytic stages of Eimeria in the small intestinal mucosa (Stained with toluidine blue; magnification: $\mathrm{x} 400$ (A) and x1000 (B))
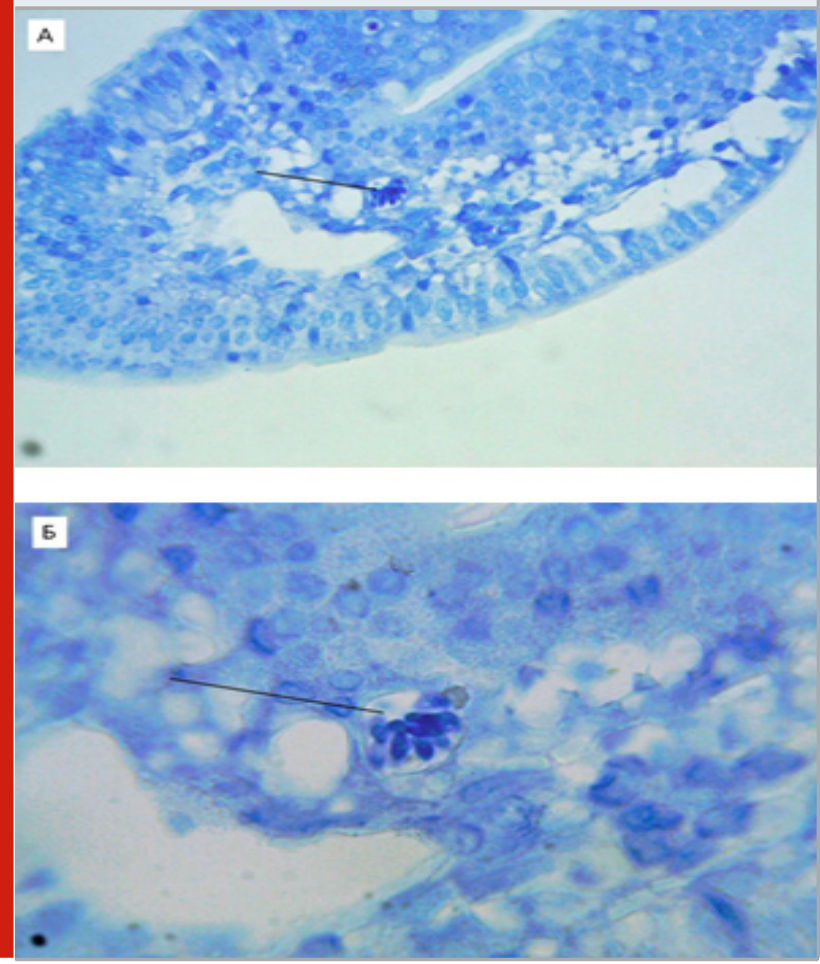

Merozoites, as part of the meronts, are clearly visible and easily differentiated from fibroblastic cells and nuclei of caemic enterocytes (Figure 2). When stained with Gill's haematoxylin and alcohol eosin (Figure 3) and Mayer's haematoxylin and alcohol eosin (Figure 4), the histomap is less contrasting. Merozoites within the meronte can only be differentiated by the specific shape of the parasite, while the intensity of the staining is usually hardly differentiated from the nuclei of enterocytes and fibroblasts of the mucous membrane's own plate. The least informative, in our opinion, is Van Gieson staining (Figure 5) (Sidorenko et al., 2020).

As can be seen in Figure 5, there is no contrast: the cell nuclei differ faintly in colour from the cytoplasm and it becomes very difficult to detect merozoites within the meronts. The small intestine wall of the experimental animals retains its typical structure. 
Figure 3. Damage caused by endocytic stages of Eimeria in the epithelial plate of the small intestinal mucosa (Staining: Gill's haematoxylin and 1\% alcohol eosin; magnification: $x 400(\mathrm{~A})$ and x1000 (B)

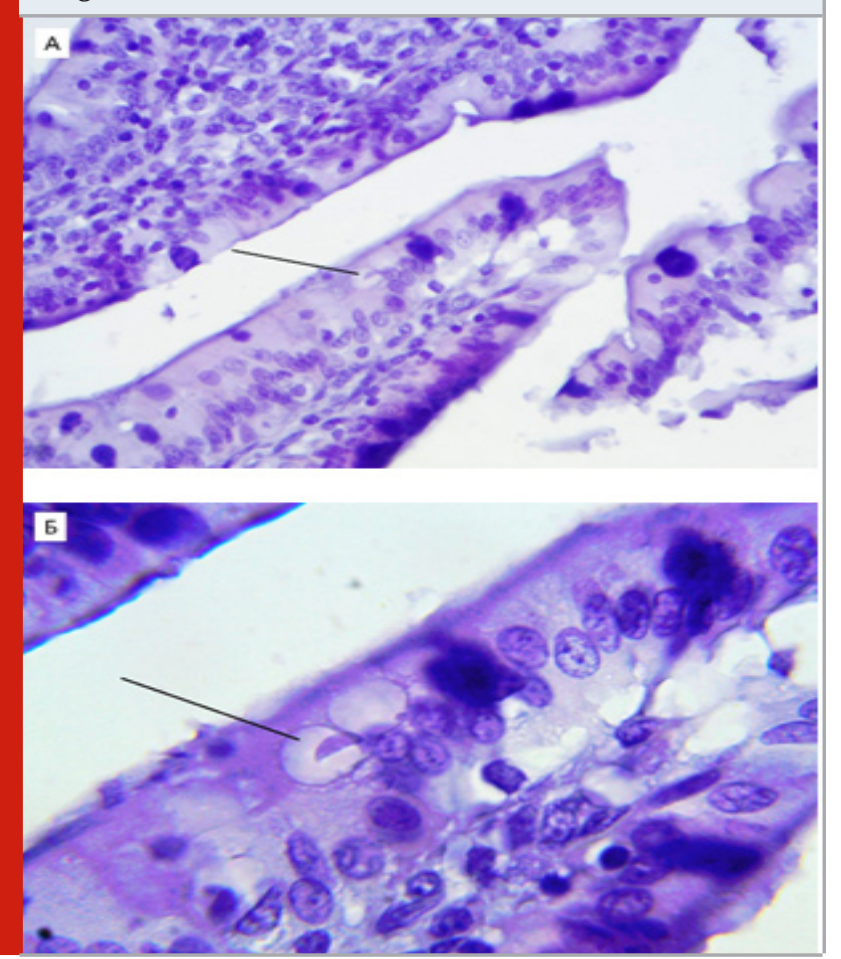

Figure 4. Damage caused by endocytic stages of Eimeria in the epithelial and intrinsic plate of the small intestinal mucosa (Staining: Mayer's haematoxylin and 1\% alcohol eosin; magnification: $x 400(\mathrm{~A})$ and $\mathrm{x} 1000$ (B)
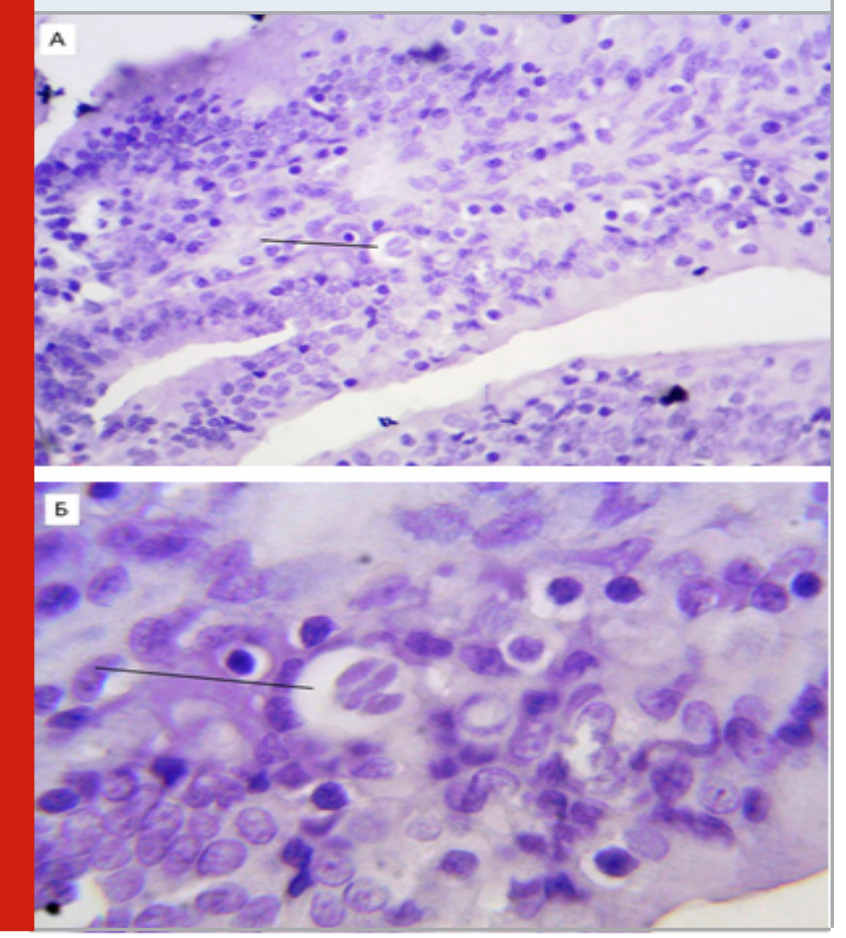

Figure 5. Damage caused by endocytic stages of Eimeridae in

epithelial and intrinsic mucosal plate of the small intestine (Stains: Perls van Gieson; magnification: x400 (A) and $\mathrm{X} 1000(\mathrm{~B}))$
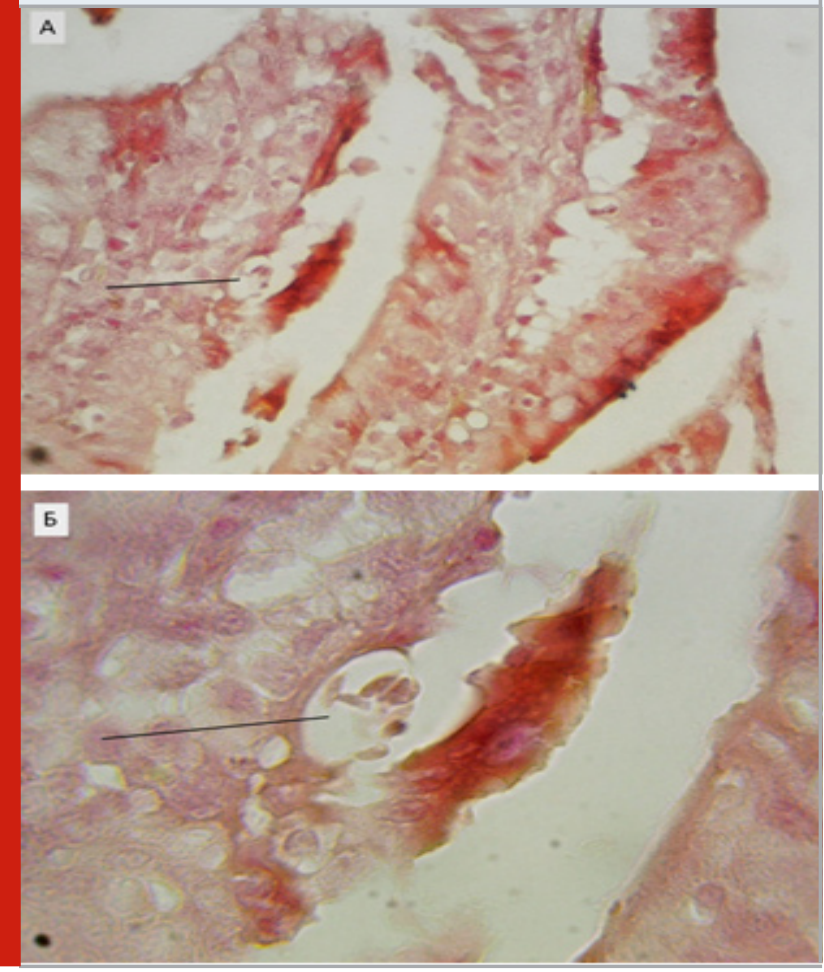

Enterocytes of the apical part of some villi show signs of intracellular edema accompanied by fusion of cell borders, moderate lucidity of the cytoplasm and nucleus, and smoothing of the chromatin pattern. There is often an increase in secreted bocalytic cell activity in the form of increased intracellular vacuole size.

Intracellular large-volume vacuolisation of other enterocytes is not uncommon, mainly on the basal pole side of the enterocytes. In addition, parasitiform vacuoles formed by eimeria are found in the structure of the mucosa. At the basal lamina level, multiple small to medium-sized cavities can be observed in the structure of many villi, which may indicate subepithelial oedema. The stromal part of the intestinal villi sometimes shows a similar pathological pattern. The mucosa is diffusely infiltrated with small lymphocytes, which subepithelially form linear dense clusters (Sokół et al., 2014).

\section{CONCLUSION}

When determining the intensity of infestation in different breed and age groups, it was found that Californian animals were most susceptible to infestation by $\mathrm{E}$. perforans and E. irresidua oocysts, regardless of the dose of infestation. The histological examination of the small intestine of infected rabbits of experimental breed-age groups revealed that despite the absence of any clinical manifestations, damage to the epithelial plate of the small intestine mucosa was found in the epithelial plate 
of the intestinal villi as well as significant damage in the intrinsic plate of the mucosa, which are most effectively revealed by staining with alcyan blue and Carazzi hematoxylin and toluidine blue.

Source of funding: The authors declare that they have no funding support for this study.

Conflict of interest: The authors declare that there is no known conflict of interest associated with this publication.

\section{REFERENCES}

Edwards, E. E., Garner, B. C., Williamson, L. H., Storey, B. E., Sakamoto, K. (2016). Pathology of Haemonchus contortus in New World camelids in the southeastern United States: A retrospective review. Journal of Veterinary Diagnostic Investigation, 28(2), 105-109.

Ilic, T., Stepanovic, P., Nenadovic, K., Dimitrijevic, S. (2018). Improving agricultural production of domestic rabbits in Serbia by follow-up study of their parasitic infections. Iranian Journal of Veterinary Research, Shiraz University, 19 (4), 290-297.

Kuznetsov Yu.E., Belova L.M., Gavrilova N.A. (2018). Evaluation of the effectiveness of drugs for mink eimeriosis, Indo American Journal of Pharmaceutical Sciences. 6(3): 6849-6854.
Mykhailiutenko, S. M., Kruchynenko, O. V., Klymenko, O. S., Serdioucov, J. K., Dmytrenko, N. I., Tkachenko, V. V. (2019). Pathomorphological changes in the large intestine of rabbits parasitised by Passalurus ambiguus (Nematoda, Oxyuridae). Regulatory Mechanisms in Biosystems, 10(1), 69-74.

Ola-Fadunsin, S. D., \& Ibitoye, E. B. (2017). A retrospective evaluation of parasitic conditions and their associated risk factors in sheep and goats in Osun state, Nigeria. Sokoto Journal of Veterinary Sciences, 15(3): 15-24.

Pehlivanoglu, B., Doganavgargil, B., Sezak, M., Nalbantoglu, i., Korkmaz, M. (2016). Gastrointestinal parasitosis: Histopathological insights to rare but intriguing lesions of the gastrointestinal tract. Türkiye Parazitoloji Dergisi, 32(2), 82-90.

Sidorenko K., Mkrtchyan M., Kuznetsov Y., Klimova E. (2020). The pathogenic effect of eimeria on rabbits of the soviet chinchilla breed and its hybrids with the Californian breed. Advances in Animal and Veterinary Sciences. 8(2): 7-11.

Sokół, R., Gesek, M., Ras-Norynska, M. and Michalczyk, M. (2014). Toltrazuril (Baycox) treatment against coccidiosis caused by Eimeria Sp. in Japanese quails (Coturnix cotur-nix Japonica). Pol. J. Vet. Sci., 17(3): 465-468 